\title{
APPROXIMATE CALCULATION METHOD OF SUBWATER PIPELINE'S FORM UNDER ITS LAYING ON SEA-BOTTOM
}

\author{
V. N. Shinkin 1 \\ ${ }^{1}$ National University of Science and Technology "MISIS" (Moscow, Russia)
}

E-mail: shinkin-korolev@yandex.ru

\section{AUTHOR'S INFO}

V. N. Shinkin, Dr. Sci. (Phys.-Math.), Prof.

\section{Key words:}

elastic line of slightly-

sloping beam, non-stretched perfectly-flexible thread,

slightly-sloping hard thread, elastic flexible rod, approximate solution, power series, subwater pipeline.

\author{
A B S T RACT
}

The various classical approximations are used to describe the shape of the subwater part of pipelines: for example, the equations of elastic line of slightly-sloping beam, non-stretched ideal flexible thread and slightly-sloping rigid thread. However, all these equations have the significant drawbacks that prevent them from being applied for the accurate calculating of the shape of subwater pipelines at their laying in deep-water seas, which are usually much deeper than lakes and rivers. So, the equations of the elastic line of slightly-sloping beam and slightly-sloping rigid thread are valid only in the case of small inclination angles of the pipeline, and the equation of the non-stretched ideal flexible thread (the equation of the chain line of the cable) does not take into account the resistance of the pipeline to bending and torsion. The subwater main pipelines are laid on the sea-bottom by the special marine ships and can have a laying depth of more than two kilometers. In this case, the longitudinal axis of the pipeline is so much curved during the laying that the excessive bending can lead to the destruction or defects of the pipeline. Therefore, at the laying of pipelines, it is extremely important to know the shape of their free subwater part in water and to correctly apply the pontoons, stingers and pipeline tension with the help of the anchors of the marine ships. The nonlinear equation of fourth order for the elastic flexible rod does not involve restrictions on the inclination angle of the subwater part of the pipeline and takes into account the resistance of the pipeline to bending. Although this nonlinear equation has no the general analytical solution, it can be solved numerically, using the Runge-Kutta method of fourth-order accuracy, or approximately by means of the power series. The approximate solution of the equation of elastic flexible rod by means of a power series is given below. For this solution, the coefficients of the series are found up to the 12th degree of the series argument. As an example of the use of the found approximate solution, the numerical calculation of the shape and inclination angle of the subwater part of TurkStream at its laying on the sea-bottom are given.

\section{Introduction}

The Russian subwater main pipelines play a strategic role in the development of Russia's energy engineering and economy. The main pipelines consist of a large number of the thick-walled steel pipes of large diameter welded together. So, the subwater part of TurkStream, consisting of two parallel threads (about $930 \mathrm{~km}$ long each), runs on the sea-bottom and consists of 155,000 pipes. With a full load, the capacity of TurkStream is 31.5 billion $\mathrm{m}^{3}$ of gas per year.

When laying pipelines from the decks of the specialized marine ships (from the sea surface) to the seabottom, the pipeline experiences the significant external power loads, under the influence of which there is a strong bending of its axis [1-14]. If the bend is too large, then the irreversible plastic deformations (defects in the shape of the pipeline) occur on the outer surface of the pipeline wall, which lead to the irreversible curvatures of the pipeline axis, loss of the cylindrical shape of the pipes and even destruction of the pipe walls. To reduce the curvature of the longitudinal axis of the subwater part of the pipelines, the various devices are used: the unloading buoys and pontoons, the stingers and so on. However, under a sufficient sea-bottom depth, these devices can only be used in a limited area of the subwater part of the pipeline. Therefore, a significant part of the subwater pipeline is in a free subwater state and experiences only the gravitational force of the Earth's gravity, the Archimedean buoyancy force and the longitudinal tension, created by the marine ship with the help of the special anchors.

(c) SHINKIN V. N., 2019
The especially strong curvatures of the pipeline axis are observed near the point of its separation from the sea-bottom and near the point of its separation from the stinger, located at the end of the marine ship (Fig. 1). In order to calculate the curvature of the subwater part of the pipeline, it is necessary to know the equation of its elastic line and the boundary conditions at both ends of the subwater part of the pipeline.

\section{Classical equations for pipeline's shape}

The equation of elastic line of slightly-sloping beam has the form [1-6]

$$
E J \frac{d^{2} y}{d x^{2}}=M, \quad \frac{d^{4} y}{d x^{4}}+\frac{q}{E J}=0,\left(\frac{d y}{d x}\right)^{2}<<1,|y|<<1,
$$

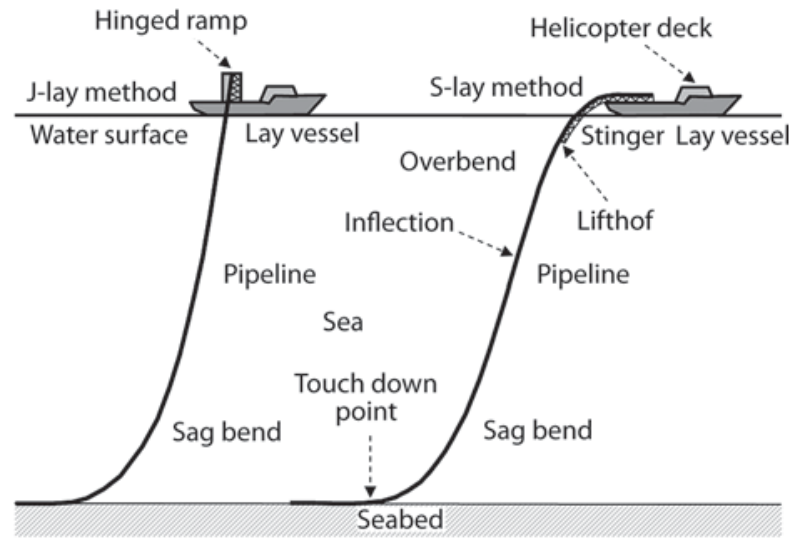

Fig. 1. Pipeline's laying on sea-bottom 
where $E$ is the young's modulus, $J$ and $M$ are the axial moment of inertia and bending moment of the beam's cross-section, $q$ is the linear weight of the beam, $y$ is the vertical deflection of the beam (the horizontal axis $x$ is directed along the axis of the beam).

The general solution of the equation of elastic line of slightly-sloping beam has the form

$$
\begin{aligned}
& y(x)=C_{0}+C_{1} x+\frac{1}{2} C_{2} x^{2}+\frac{1}{6} C_{3} x^{3}-\frac{1}{24} \frac{q}{E J} x^{4} \\
& C_{i}=\text { const }, \quad i=0,1,2,3 .
\end{aligned}
$$

To describe the shape of the pipeline, this equation is not suitable, since its derivation assumes that all external force factors (the concentrated forces, distributed loads and support reactions) are strictly perpendicular to the axis of the beam, and the deflection of the beam $y$ is very small.

A thread that does not resist bending and torsion is called an ideal flexible thread.

The equation of non-stretched ideal flexible thread (the equation of chain line of cable) has the form [3]

$$
H \frac{\mathrm{d}^{2} y}{\mathrm{~d} x^{2}}-q \sqrt{1+\left(\frac{\mathrm{d} y}{\mathrm{~d} x}\right)^{2}}=0, \quad M=0,
$$

where $q$ is the linear weight of thread, $H$ is the horizontal tractive force (of marine ship), $x$ is the horizontal axis.

The general solution of the equation of ideal flexible thread has the form

$$
\begin{aligned}
& y=a \operatorname{ch}\left(\frac{x-C_{1}}{a}\right)+C_{2}, \quad a=\frac{H}{q}=\text { const, } \\
& C_{i}=\text { const }, \quad i=1,2 ;
\end{aligned}
$$

where the function ch is a hyperbolic cosine.

To describe the shape of the pipeline, this equation is also not suitable, since its derivation we neglect the bending resistance of the pipe (we neglect the bending moment $M$ of the cross-section of the pipe). At the zero boundary conditions $(y(0)=0, d y(0) / d x(0)=0)$, the curvature of the longitudinal axis of the thread at the point of separation from the horizontal surface $(x=0)$ is not zero: $\varepsilon(0)=q / H \neq 0$. For the subwater pipelines, the curvature $\varepsilon(0)=0$.

A slightly-sloping thread is a thread of relatively small curvature, the square of the tangent of the inclination angle of which is much less than one.

The equation of slightly-sloping rigid thread has the form $[1,2,4]$

$$
\frac{\mathrm{d}^{4} y}{\mathrm{~d} x^{4}}-\frac{H}{E J} \frac{\mathrm{d}^{2} y}{\mathrm{~d} x^{2}}+\frac{q}{E J}=0,\left(\frac{\mathrm{d} y}{\mathrm{~d} x}\right)^{2} \ll<1, \quad M=E J \frac{d^{2} y}{d x^{2}},
$$

and used to describe the shape of the slightly-sloping pipeline.

The general solution of the equation of slightly-sloping rigid thread has the form

$$
\begin{aligned}
& y(x)=C_{1} \operatorname{ch} k x+C_{2} \operatorname{sh} k x+\frac{q}{H} \frac{x^{2}}{2}+C_{3} x+C_{4}, \\
& k=\sqrt{\frac{H}{E J}} ; \quad C_{i}=\text { const }, \quad i=1,2,3,4 .
\end{aligned}
$$

The equation of elastic flexible rod has the form

$$
\frac{\mathrm{d}^{2}}{\mathrm{~d} x^{2}}\left(\frac{\frac{\mathrm{d}^{2} y}{\mathrm{~d} x^{2}}}{\left(1+\left(\frac{\mathrm{d} y}{\mathrm{~d} x}\right)^{2}\right)^{3 / 2}}\right)-\frac{H}{E J} \frac{\mathrm{d}^{2} y}{\mathrm{~d} x^{2}}+\frac{q}{E J} \sqrt{1+\left(\frac{\mathrm{d} y}{\mathrm{~d} x}\right)^{2}}=0 .
$$

The derivation of this equation takes into account the bending moment of the cross-section of the rod (pipe) and does not impose any restrictions on the first derivative $d y / d x$.

There is no analytical solution of this equation, so it has to be solved either numerically or approximately.

\section{Approximate solution of equation of elastic flexible rod}

The boundary conditions of the problem (at subwater laying of the pipeline) at the left end of the elastic flexible rod (pipeline) have the form

$$
y(0)=0, \quad \frac{d y}{d x}(0)=0, \quad \frac{d^{2} y}{d x^{2}}(0)=0 .
$$

Under the given zero boundary conditions, the analytical solution of the equation of elastic flexible rod in the form of a power series by $x$ has the form

$$
\begin{aligned}
& y(x)=a_{3} x^{3}+a_{4} x^{4}+a_{5} x^{5}+a_{6} x^{6}+a_{7} x^{7}+ \\
& +a_{8} x^{8}+a_{9} x^{9}+a_{10} x^{10}+a_{11} x^{11}+a_{12} x^{12}+\ldots, \\
& a_{3}=-\frac{1}{6} C_{3} \frac{H}{E J_{x}}, \quad a_{4}=-\frac{1}{24} \frac{q}{H} \frac{H}{E J_{x}}, \\
& a_{5}=-\frac{1}{120} C_{3}\left(\frac{H}{E J_{x}}\right)^{2}, \quad a_{6}=-\frac{1}{720} \frac{q}{H}\left(\frac{H}{E J_{x}}\right)^{2}, \\
& a_{7}=-C_{3}\left[\frac{1}{5040}+\frac{1}{112} C_{3}^{2}\right]\left(\frac{H}{E J_{x}}\right)^{3}, \\
& a_{8}=-\left[\frac{1}{40320}+\frac{53}{6720} C_{3}^{2}\right] \frac{q}{H}\left(\frac{H}{E J_{x}}\right)^{3}, \\
& a_{9}=-C_{3}\left[\frac{1}{362880}+C_{3}^{2} \frac{5}{2688}\right]\left(\frac{H}{E J_{x}}\right)^{4}- \\
& -\frac{85}{36288} C_{3}\left(\frac{q}{H}\right)^{2}\left(\frac{H}{E J_{x}}\right)^{3}, \\
& a_{10}=-\left[\frac{1}{3628800}+\frac{583}{403200} C_{3}^{2}\right] \frac{q}{H}\left(\frac{H}{E J_{x}}\right)^{4}- \\
& -\frac{17}{72576}\left(\frac{q}{H}\right)^{3}\left(\frac{H}{E J_{x}}\right)^{3}, \\
& a_{11}=-C_{3}\left(\frac{2461}{6652800}\left(\frac{q}{H}\right)^{2}+\frac{1}{39916800} \frac{H}{E J_{x}}\right)\left(\frac{H}{E J_{x}}\right)^{4}-
\end{aligned}
$$




$$
\begin{gathered}
-\frac{75913}{479001600} C_{3}^{3}\left(\frac{H}{E J_{x}}\right)^{5}+\frac{227}{10644480} C_{3}{ }^{5}\left(\frac{H}{E J_{x}}\right)^{5}, \\
a_{12}=-\left(\frac{2461}{79833600}\left(\frac{q}{H}\right)^{2}+\frac{1}{479001600} \frac{H}{E J_{x}}\right)\left(\frac{q}{H}\right)\left(\frac{H}{E J_{x}}\right)^{4}- \\
-\frac{1339}{10644480} C_{3}{ }^{2}\left(\frac{q}{H}\right)\left(\frac{H}{E J_{x}}\right)^{5}-\frac{731}{443520} C_{3}{ }^{4}\left(\frac{q}{H}\right)\left(\frac{H}{E J_{x}}\right)^{5} .
\end{gathered}
$$

where $C_{3}$ - is a dimensionless constant to be determined from the boundary conditions at the right end of the subwater part of the pipeline at the point of contact with the stinger.

Note that the coefficients of the expansion of the solutions of the equation of slightly-sloping rigid thread and the equation of elastic flexible rod in the power series by $x$ under the given zero boundary conditions at the left end of the rod coincide to the sixth degree of $x$ inclusive, but from the seventh degree of $x$ and above differ significantly.

The power series's coefficients for the solution of the equation of elastic flexible rod can also be determined using the recursive formulas:

$$
\begin{aligned}
& 24 a_{4}=-\frac{q}{E J_{x}}, \quad 120 a_{5}=6 \frac{H}{E J_{x}} a_{3}, \\
& 360 a_{6}=12 \frac{H}{E J_{x}} a_{4}, \quad 840 a_{7}=1620 a_{3}^{3}+20 \frac{H}{E J_{x}} a_{5}, \\
& 1680 a_{8}=30 \frac{H}{E J_{x}} a_{6}+11016 a_{3}{ }^{2} a_{4}-18 \frac{q}{E J_{x}} a_{3}^{2}, \\
& 3024 a_{9}=\frac{H}{E J_{x}}\left(42 a_{7}+81 a_{3}^{3}\right)+23328 a_{3} a_{4}{ }^{2}+ \\
& +21060 a_{3}{ }^{2} a_{5}-48 \frac{q}{E J_{x}} a_{3} a_{4}, \\
& 5040 a_{10}=\frac{H}{E J_{x}}\left(56 a_{8}+378 a_{3}{ }^{2} a_{4}\right)+35964 a_{3}{ }^{2} a_{6}+ \\
& +85320 a_{3} a_{4} a_{5}+15552 a_{4}{ }^{3}-\frac{q}{E J_{x}}\left(60 a_{3} a_{5}+32 a_{4}{ }^{2}\right), \\
& 7920 a_{11}=141264 a_{3} a_{4} a_{6}+82080 a_{4}{ }^{2} a_{5}+ \\
& +75600 a_{3} a_{5}{ }^{2}+21999 a_{3}{ }^{2} a_{7}-43740 a_{3}{ }^{5}+ \\
& +72 \frac{H}{E J_{x}} a_{9}+576 \frac{H}{E J_{x}} a_{3} a_{4}{ }^{2}+540 \frac{H}{E J_{x}} a_{3}{ }^{2} a_{5}- \\
& +72 \frac{q}{E J_{x}} a_{3} a_{6}-80 \frac{q}{E J_{x}} a_{4} a_{5}, \\
& +11880 a_{12}=244620 a_{3} a_{5} a_{6}+217728 a_{3} a_{4} a_{7}+ \\
& +140400 a_{4} a_{5}{ }^{2}+84240 a_{3}{ }^{2} a_{8}+132192 a_{4}{ }^{2} a_{6}- \\
& -390744 a_{3}{ }^{4} a_{4}+90 \frac{H}{E J_{x}} a_{10}+729 \frac{H}{E J_{x}} a_{3}{ }^{2} a_{6} a_{4} a_{5}+288 \frac{H}{E J_{x}} a_{4}{ }^{3}-
\end{aligned}
$$

$$
-81 \frac{q}{E J_{x}} a_{3}{ }^{4}-84 \frac{q}{E J_{x}} a_{3} a_{7}-50 \frac{q}{E J_{x}} a_{5}{ }^{2}-96 \frac{q}{E J_{x}} a_{4} a_{6} .
$$

where the constant $a_{3}$ is to be determined from the boundary conditions at the right end of the subwater part of the pipeline.

\section{Linear weight of concreted pipe in water}

For the subwater part of TurkStream are used pipes from the high-strength manganese-carbon steel with the length $L_{t}=12 \mathrm{~m}$, diameter $D_{t}=81.3 \mathrm{~cm}$, thickness $h_{t}=39 \mathrm{~cm}$, weight about $P_{\mathrm{t}}=90 \mathrm{kH}$ each. The pipes have a thin internal anti-friction epoxy coating and an external three-layer polypropylene coating for a corrosion protection with the thickness $h_{i s}=3 \mathrm{~mm}(2.0-3.5 \mathrm{~mm})$. The outer surface of the pipe is also covered with a small layer of concrete with the thickness $h_{\text {conc }}=6 \mathrm{~cm}(5-8 \mathrm{~cm})$ for a protection from sea water and mechanical influences.

Let $\rho_{s t} \approx 7874 \mathrm{~kg} / \mathrm{m}^{3}$ is the density of steel, $\rho_{\text {is }} \approx 920 \mathrm{~kg} / \mathrm{m}^{3}$ is the density of isolation (polypropylene), $\rho_{\text {conc }} \approx 1800 \mathrm{~kg} / \mathrm{m}^{3}$ is the density of the concrete, $\rho_{\mathrm{H}_{2} \mathrm{O}}=1000 \mathrm{~kg} / \mathrm{m}^{3}$ is the density of water. Then the linear weight $q$ and the axial moment of inertia $J$ of the pipe are equal

$$
\begin{aligned}
& P_{s t}=\frac{\rho_{s t} g \pi}{4}\left(D_{t}^{2}-\left(D_{t}-2 h_{t}\right)^{2}\right) L_{t}, \\
& P_{i s}=\frac{\rho_{i s} g \pi}{4}\left(\left(D_{t}+2 h_{i s}\right)^{2}-D_{t}^{2}\right) L_{t}, \\
& P_{\text {conc }}=\frac{\rho_{\text {conc }} g \pi}{4}\left(\left(D_{t}+2 h_{i s}+2 h_{\text {conc }}\right)^{2}-\left(D_{t}+2 h_{i s}\right)^{2}\right) L_{t}, \\
& P_{\mathrm{H}_{2} \mathrm{O}}=\frac{\rho_{\mathrm{H}_{2} \mathrm{O}} g \pi}{4}\left(D_{t}+2 h_{i s}+2 h_{\text {conc }}\right)^{2} L_{t}, \\
& P_{t}=P_{s t}+P_{i s}+P_{\text {conc }}-P_{\mathrm{H}_{2} \mathrm{O}}, \\
& q=\frac{P_{t}}{L_{t}}=\frac{\pi g}{4}\left[\begin{array}{l}
\left.\rho_{s t}\left(D_{t}^{2}-\left(D_{t}-2 h_{t}\right)^{2}\right)+\rho_{\text {conc }}\left(\left(D_{t}+2 h_{i s}+2 h_{\text {conc }}\right)^{2}-\left(D_{t}+2 h_{i s}\right)^{2}-2 h_{i s}\right)^{2}\right)- \\
-\rho_{\mathrm{H}_{2} \mathrm{O}}\left(D_{t}+2 h_{i s}+2 h_{\text {conc }}\right)^{2}
\end{array}\right)+ \\
& J=\frac{\pi}{64}\left(D_{t}^{4}-\left(D_{t}-2 h_{t}\right)^{4}\right) \approx \frac{\pi D_{t}^{3} h_{t}}{8},
\end{aligned}
$$

where $P_{s t}, P_{i s}$ and $P_{\text {conc }}$ are the weight of steel, insulation and concrete in one pipe, $P_{\mathrm{H}_{2} \mathrm{O}}$ is the Archimedean buoyancy force in water acting on one pipe, $g=9.807 \mathrm{M} / \mathrm{c}^{2}$ is the acceleration of gravity on the Earth's surface.

For the pipes of the subwater part of TurkStream, the linear weight of the concreted pipes in water is $q=3.526 \mathrm{kH} / \mathrm{m}$, the axial moment of inertia of a steel tube is $J=7.119 \cdot 10^{-3} \mathrm{~m}^{4}$, the young's modulus of steel is $E \approx 2 \times 10^{11} \mathrm{~Pa}$.

Note that the linear weight of the pipe can be regulated (increased) by means of the partially or completely filling of the pipeline with sea water. 


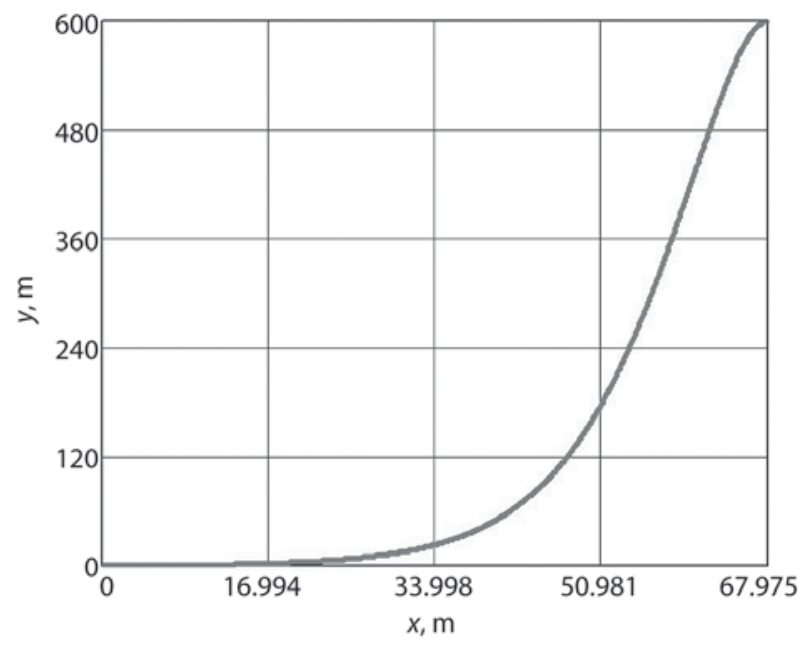

Fig. 2. Shape of subwater part of pipeline

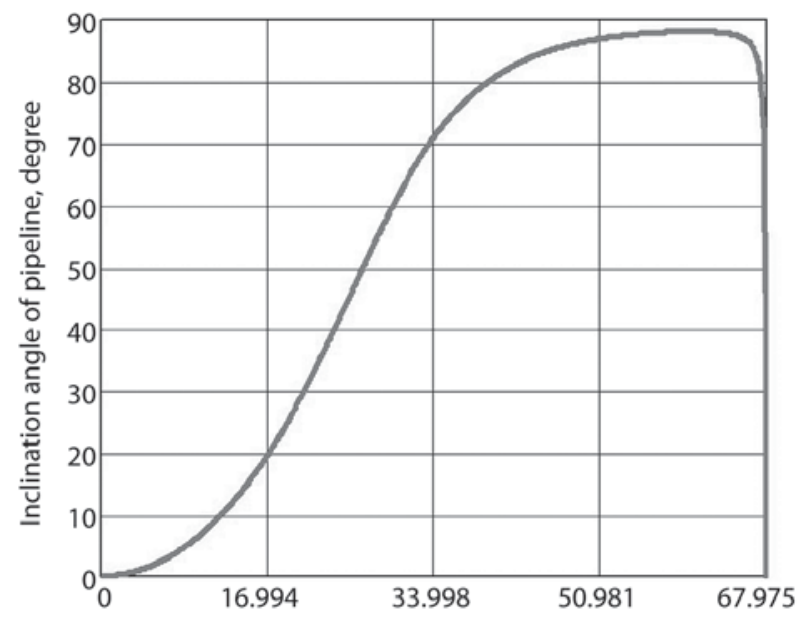

Fig. 3. Inclination angle of subwater part of pipeline

\section{Numerical calculations}

Consider the $S$-method of the subwater laying of pipes of TurkStream by means of a stinger on the sea-bottom. Let the inclination angle of the pipeline at the point of contact with the stinger $\alpha=0^{\circ}$, the sea depth at the place of the laying of the pipeline is $h=600 \mathrm{~m}$, the horizontal tractive force of the marine ship is $H=1.6 \mathrm{MH}$.

The boundary conditions of the problem at the left and right ends of the pipeline have the form

$$
\begin{aligned}
& y(0)=0, \quad \frac{d y}{d x}(0)=0, \quad \frac{d^{2} y}{d x^{2}}(0)=0 ; \\
& y(l)=h, \quad \frac{d y}{d x}(l)=\operatorname{tg} \alpha,
\end{aligned}
$$

where $\alpha$ is the inclination angle of the pipeline at point of its contact with the stinger, $l$ is the length of the projection of the subwater part of the pipeline on the horizontal sea-bottom from the point of its separation from the seabottom to the point of its touching with the stinger of the marine ship.
Substituting the approximate solution of the problem in the form of a power series by $x$ (containing the terms of the series up to 12 degrees by $x$ inclusively) into the boundary conditions at the right end of the pipeline, we find the constant $C_{3}=-2.048$ and the length $l=67.975 \mathrm{~m}$. Substituting $C_{3}$ into an approximate solution of the problem, we find the shape of the pipeline under the given boundary conditions. The shape and inclination angle of the subwater part of the pipeline (at laying pipes on the sea-bottom) are shown in Fig. 2 and Fig. 3.

\section{Conclusions}

The approximate analytical solution of the nonlinear equation of elastic flexible rod (pipeline) in the form of a power series is found, taking into account the terms of the series up to the 12th degree by the series argument. The approximate solution is used to the construction of the shape of the subwater part of TurkStream at its laying on the sea-bottom from the marine ship.

\section{REFERENCES}

1. Borodavkin P. P., Berezin V. L., Shadrin O. B. Underwater pipelines. Moscow : Nedra. 1975. 415 p.

2. Kachurin V. K. Theory of hanging systems. Static calculation. Leningrad; Moscow : Gosstroiizdat. 1962. 223 p.

3. Merkin D. R. Introduction to mechanics of flexible thread. Moscow : Nauka. 1980. 240 p.

4. Svetlitskiy V. A. Mechanics of flexible rods and threads. Moscow : Mashinostroenie. 1978. 222 p.

5. Shinkin V. N. Springback coefficient of the main pipelines' steel large-diameter pipes under elastoplastic bending. CIS Iron and Steel Review. 2017. Vol. 14. pp. 28-33.

6. Shinkin V. N. Arithmetical method of calculation of power parameters of $2 \mathrm{~N}$-roller straightening machine under flattening of steel sheet. CIS Iron and Steel Review. 2017. Vol. 14. pp. 22-27.

7. Zorin E. E., Lanchakov G. A., Stepanenko A. I., Shibnev A. V. Serviceability of pipelines. Part 1. Calculared and operational reliability. Moscow : Nedra. 2000. 244 p.

8. Lanchakov G. A., Zorin E. E., Pashkov Ju. I., Stepanenko A. I. Serviceability of pipelines. Part 2. Resistance to destruction. Moscow : Nedra. 2001. 350 p.

9. Lanchakov G. A., Zorin E. E., Stepanenko A. I. Serviceability of pipelines. Part 3. Diagnostics and prediction of resource. Moscow : Nedra. 2003. 291 p.

10. Shinkin V. N. Simplified calculation of the bending torques of steel sheet and the roller reaction in a straightening machine. Steel in Translation. 2017. Vol. 47. No. 10. pp. 639-644.

11. Mazur I. I., Ivantsov O. M. Safety of pipeline systems. Moscow : ELIMA. 2004. 1104 p.

12. Demchenko V. G., Demchenko G. V. Arterial pipelines. Reliability. Conditions of work and destructions. Moscow : Nedra. 2007. $304 \mathrm{p}$.

13. Koroshak A. A., Nechval A. M. Designing and exploitation of oil-and-gas pipelines. Saint Petersburg : Nedra. 2008. 488 p.

14. Tetelmin V. V., Yazev V. A. Main oil-and-gas pipelines. Dolgoprudny: Intellekt. 2010. 352 p. 